\title{
Finite element analysis of stress distribution in four different endodontic post systems in a model canine
}

\author{
Aijie Chen, Xiaoli Feng, Yanli Zhang, Ruoyu Liu and Longquan Shao* \\ Nanfang Hospital, Southern Medical University, Guangzhou 510515, China
}

\begin{abstract}
To investigate the stress distribution in a maxillary canine restored with each of four different post systems at different levels of alveolar bone loss. Two-dimensional finite element analysis (FEA) was performed by modeling a severely damaged canine with four different post systems: CAD/CAM zirconia, CAD/CAM glass fiber, cast titanium, and cast gold. A force of $100 \mathrm{~N}$ was applied to the crown, and the von Mises stresses were obtained. FEA revealed that the CAD/CAM zirconia post system produced the lowest maximum von Mises stress in the dentin layer at $115.8 \mathrm{MPa}$, while the CAD/CAM glass fiber post produced the highest stress in the dentin at 518.2 MPa. For a severely damaged anterior tooth, a zirconia post system is the best choice while a cast gold post ranks second. The CAD/CAM glass fiber post is least recommended in terms of stress level in the dentin.
\end{abstract}

Keywords: FEA, CAD/CAM, post, canine

\section{Introduction}

With increasing demand for tooth retention, fixed prostheses have faced great challenges in the case of teeth that have been damaged by caries [1], fracture [2], excessive preparation, or idiopathic reasons. Several factors that influence the strength of endodontically treated teeth have been previously discussed [3].

The loss of alveolar bone around the root greatly influences tooth strength [4]. An in vitro study [5] showed that loss of alveolar bone mass is a major reason for the failure of restoration of the post and core. Some studies [6] also found that teeth treated endodontically had more bone loss than untreated ones in periodontitis patients. Besides alveolar bone levels, the general condition of teeth is another important factor in their strength. Excessive preparation of the root canal may reduce the strength of the root dentin walls, which significantly decreases their fracture resistance [7]. When the coronal tooth structure has been reduced to less than half, posts are needed to restore the strength [8].

The survival of the restoration of severely damaged teeth depends on the design of the posts [9]. Some studies $[10,11]$ suggest that the supporting dowels (or posts) should have elastic moduli close to that of the natural tooth, which reduces the stress concentration and thus the rate of failure. As shown

\footnotetext{
* Address for correspondence: Longquan Shao, Nanfang Hospital, Southern Medical University, Guangzhou 510515, China. Tel.: +86 15989283921; Fax: 020-62787153; E-mail: shaolongquan@smu.edu.cn.
} 
in Table 1, the elastic modulus of glass fiber $(20 \mathrm{GPa})$ is very close to that of dentin (18.6 GPa). However, the diameters of fiber posts cannot be completely filled with the prepared space. Moreover, there is a weak interface due to the separated composite resin core [12]. Toksavul, et al. [13] stated that zirconia post systems produced slightly lower stress concentrations in dentin than glass fiber and titanium posts.

The purpose of our study was to use the finite element method (FEM) to investigate the stress distribution of severely damaged maxillary anterior canines with different levels of alveolar bone loss, restored with four different post systems.

\section{Materials and methods}

The two-dimensional FEM structural analysis program, ABAQUS 6.6, (SIMULIA, Providence, RI, USA) was used in our study. The sample tooth was obtained from the Department of Anatomy of Southern Medical University (Guangzhou, China), and was selected in compliance with the standard criteria for an average Chinese human tooth.

\subsection{Finite element model}

Forty-eight models were constructed based on the measurements of the labiolingual cross-sectional anatomy of the sample. The models varied in their alveolar bone level, cervical dentin wall thickness, and post materials. According to clinical standards, all numerical models were designed to represent an endodontically treated maxillary canine restored with a post and a lithia disilicate-based glass ceramic crown. Certain modifications were made to maintain geometric symmetry and avoid sharp corners.

Table 1

Mechanical properties of dental structures and materials

\begin{tabular}{lll}
\hline Material & Elastic modulus $(\mathrm{GPa})$ & Poisson's ratio \\
\hline Dentin & 18.6 & 0.31 \\
Gutta-percha & 0.00069 & 0.45 \\
Glass fiber post & 20 & 0.30 \\
Glass ceramic & 62 & 0.30 \\
Zirconia & 200 & 0.33 \\
Gold & 96.6 & 0.35 \\
Titanium & 210 & 0.33 \\
\hline
\end{tabular}
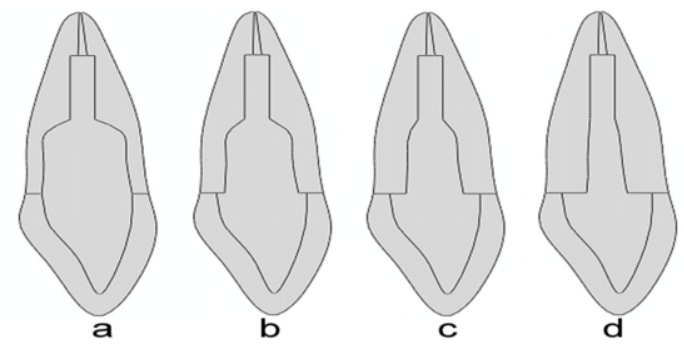

Fig. 1. Models with different dentin wall thickness: a. $1 \mathrm{~mm}$, b. $1.5 \mathrm{~mm}$, c. $2 \mathrm{~mm}$, d. $2.5 \mathrm{~mm}$.
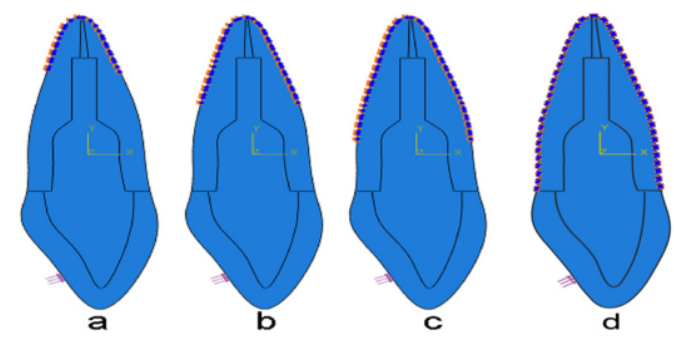

Fig. 2. Models with different boundary conditions simulating alveolar bone loss: a. 1/3 bone level, b. 1/2 bone level, c. 2/3 bone level, d. no bone loss. 
The root canal space was prepared with four different thicknesses of dentin walls: $1.0 \mathrm{~mm}, 1.5 \mathrm{~mm}$, $2.0 \mathrm{~mm}$, and $2.5 \mathrm{~mm}$ (Figure 1). Four different systems of posts and cores were considered in this study, including computer-aided designed/computer-aided manufactured (CAD/CAM) zirconia, $\mathrm{CAD} / \mathrm{CAM}$ glass fiber, cast titanium, and cast gold. The CAD/CAM or cast posts were customized for the root canal. Moreover, alveolar bone levels were simulated as horizontal bone loss of a. $1 / 3$ bone level, b. $1 / 2$ bone level, c. $2 / 3$ bone level, and d. no bone loss (Figure 2). For simplification purposes, alveolar bone loss levels were simulated as boundary constraints.

\subsection{Stress analysis using FEA}

Mesh convergence testing was employed in our study, and the average diameter in ideal element size was $0.2 \mathrm{~mm}$. The model was meshed with 3 degrees of freedom per node, resulting in 6296 elements and 3267 nodes. The elastic properties of the materials [14] are shown in Table 1. A force of $100 \mathrm{~N}$ was applied $3 \mathrm{~mm}$ below the incisal edge of the crown at a $45^{\circ}$ angle [15]. The maximum von Mises stresses were calculated under different conditions and in different regions.

\section{Results}

The maximum stress values are shown in Figures 3-6. Table 2 and Figure 6 present the maximum equivalent stresses in the dentin, post, and crown for different conditions. In Figure 3, there was stress concentration between the post and the root dentin in the CAD/CAM zirconia model, and significant differences were observed among the four dentin thickness conditions. The group with $1.5 \mathrm{~mm}$ thickness showed the highest maximum Mises stress of 271.0 MPa in dentin; however, the stress of this group at the post/root interface was slightly lower than that in the other three groups.

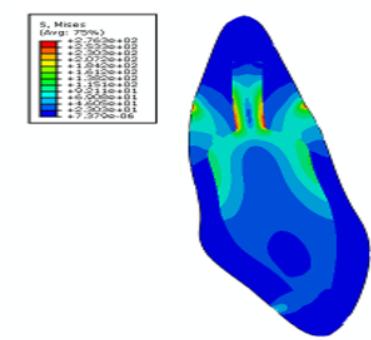

(a)

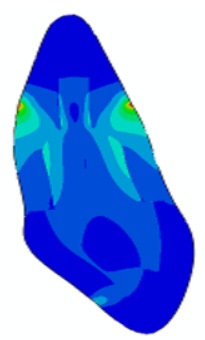

(b)

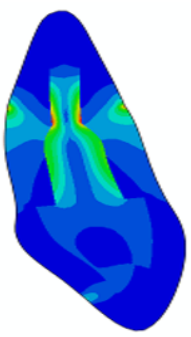

(c)

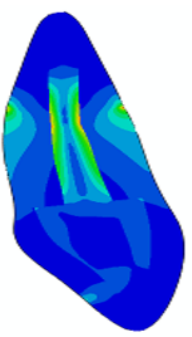

(d)

Fig. 3. Stress distribution in the zirconia post system with different dentin thicknesses under boundary conditions of 1/2 bone level: a. $1.0 \mathrm{~mm}$, b. $1.5 \mathrm{~mm}$, c. $2.0 \mathrm{~mm}$, d. $2.5 \mathrm{~mm}$.

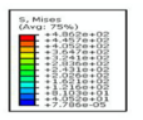

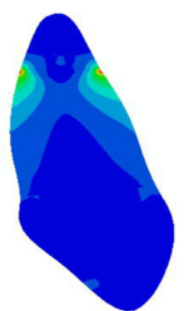

(a)

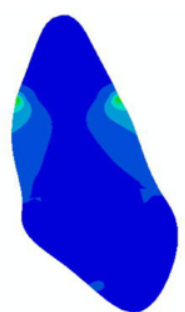

(b)

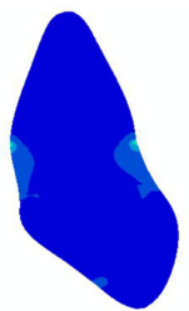

(c)

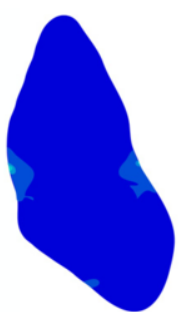

(d)

Fig. 4. Stress distributions in teeth with different levels of bone loss at $1 \mathrm{~mm}$ dentin thickness, a. 1/3 bone level, b. 1/2 bone level, c. $2 / 3$ bone level, d. no bone loss. 


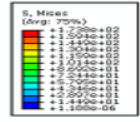

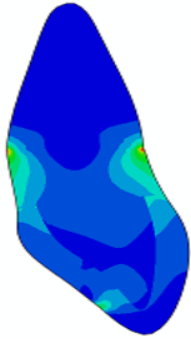

(a)

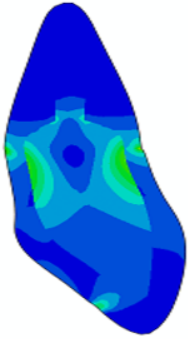

(b)

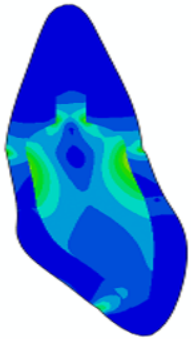

(c)

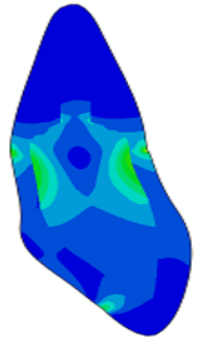

(d)

Fig. 5. Stress distributions in teeth with different post materials under $1 \mathrm{~mm}$ dentin thickness and 2/3 bone level: a. glass fiber, b. titanium, c. zirconia, d. gold.

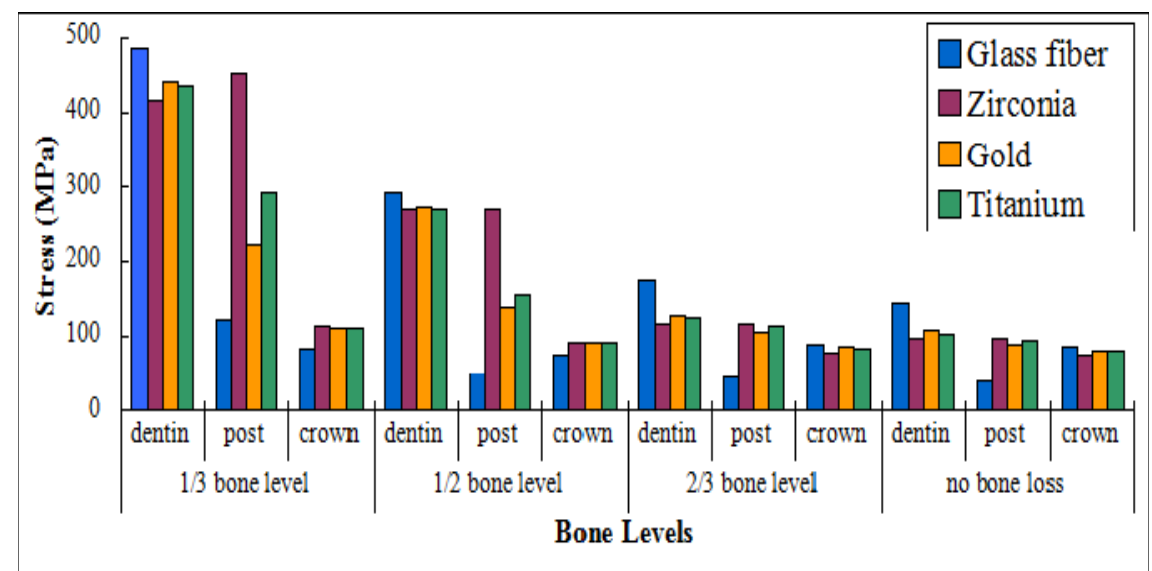

Fig. 6. Maximum von Mises stress values in different regions with various bone levels and post systems (MPa).

Table 2

Maximum von Mises stress values in different regions of gold and titanium posts with $1.5 \mathrm{~mm}$ dentin wall thickness (MPa)

\begin{tabular}{lllllll}
\hline & \multicolumn{2}{l}{ Gold $(1.5 \mathrm{~mm}$ dentin thickness $)$} & \multicolumn{3}{l}{ Titanium (1.5 mm dentin thickness) } \\
\cline { 2 - 7 } & dentin & post & crown & dentin & post & crown \\
\hline $1 / 3$ bone level & 467.5 & 116.9 & 77.9 & 463.8 & 115.9 & 77.3 \\
$1 / 2$ bone level & 280.5 & 70.1 & 93.5 & 271.7 & 90.6 & 90.6 \\
$2 / 3$ bone level & 161.4 & 53.8 & 80.7 & 158.1 & 52.7 & 79.0 \\
No bone loss & 117.5 & 44.2 & 75.1 & 128.4 & 47.3 & 75.6 \\
\hline
\end{tabular}

As shown in Figure 4, the stress distribution of the CAD/CAM glass fiber post system differed significantly depending on the bone level condition. At the bone level boundary condition with $1 / 3$ bone level of the tooth, and with a dentin thickness of $1.5 \mathrm{~mm}$, the CAD/CAM glass fiber post system displayed the highest maximum von Mises stress of $486.2 \mathrm{MPa}$ in the dentin layer. The same post system with a bone level of $2 / 3$ showed the lowest maximum von Mises stress of $173.8 \mathrm{MPa}$ in the dentin. The major stresses of the post systems were located at the cervix of the boundary constraint regions and force application areas for all models.

In terms of comparing the four different post and core materials, Figure 5 shows the differences among the four groups. The maximum von Mises stress in the glass fiber post was significantly larger than in the zirconia, and the stress concentration was primarily located in regions where the force was 
applied and the boundary constraint areas. There was no significant difference in stress distributions between the cast titanium and cast gold posts in either the post-and-core restoration or in the root dentin.

\section{Discussion}

From the FEA, the post and core material, dentin wall thickness, and alveolar bone levels all have significant effects on the von Mises stress concentrations.

\subsection{Alveolar bone level}

In this study, the CAD/CAM glass fiber post displayed a maximum von Mises stress of $486.2 \mathrm{MPa}$ in the dentin with $1 / 3$ bone level. The maximum von Mises stress increased when the alveolar bone level decreased. When keeping bone loss levels and dentin wall thickness constant, the maximum von Mises stress of the zirconia post in the dentin was lower than for other materials. With a bone level of $1 / 3$, the FEA-determined maximum stress values exceeded the yield stress limit of dentin (245 MPa [12]) for all of the post materials; this might easily lead to root fracture. In comparison to an intact tooth, teeth restored with a post and core system are reduced in strength by $21-25 \%$ [16]. Loss of $1 / 4$ or $1 / 2$ of the alveolar bone significantly reduces a tooth's loadability. However, the effect of the periodontal ligament (PDL), which can transmit occlusal force, was not considered in this study because of limitations of the laboratory conditions. More effective models of the maxillary canine accounting for fine alveolar tissue and the PDL must be created for future investigations.

\subsection{Dentin wall thickness}

Four thicknesses of dentin walls were evaluated in this study; the difference of stress values in the dentin between the four dentin thickness groups was found to be insignificant. As shown in Figure 3, the maximum stress was observed at the thickness of $1.5 \mathrm{~mm}$, while the minimum was at $2.5 \mathrm{~mm}$ thickness. The reduction of the thickness of the dentin wall may increase the possibility of fracture in the root [17]. Clinical experience has shown that tooth fracture can easily occur after several years, due to fatigue failure. This study showed that glass fiber posts generated the highest von Mises stress in the dentin wall. The shape of the root canals also influences the stress distribution within the structure of a restoration. As a result, the strength of a tooth has a close relationship to the thickness of dentin tissue around the post.

\subsection{Post and core materials}

According to the FEA, the zirconia post produced low von Mises stress in the dentin, but high stresses were observed in the post and the root dentin. The modulus of zirconia is higher than that of the hard tissue of the tooth, hence the stiffer post absorbed most of the load. Since the force was transmitted apically, the stress was easily accumulated in the root, leading to stress concentration [18]. The FEA indicated that the fiber post was not suitable for the failure of a restoration, especially in severely damaged teeth [19]. However, some previous studies [20] found no significant differences between gold alloy-based posts and glass fiber posts, while Beck [21] and Bittner [22] stated that there was no difference in the fracture load capability between prefabricated glass fiber posts and 
copy-milled zirconia posts.

\section{Conclusion}

By using the FEA method, parameters can be adjusted easily, simulations can be performed without the need for human material, and maximum standardization is possible. These FEA models are only approximations to the actual physical properties of biological structures (such as bone, tooth, and soft tissues), because the materials in the models are assumed to be homogeneous with a linear response to stress. However, in a real patient, the response to stress is more complex. The FEA used in this study is a necessary step toward later laboratory tests. Clinical studies are also necessary to acquire the longevity and success rates of post systems.

\section{Acknowledgments}

This work was supported by the National Natural Science Foundation of China (31070857, 50973045, 51172283 and 81400557), and the Project on the Integration of Industry, Education and Research of Guangdong Province, China (2012B091000147).

\section{References}

[1] P. Svanborg, L. Langstrom, R.M. Lundh, G. Bjerkstig and A. Ortorp, A 5-year retrospective study of cobalt-chromium-based fixed dental prostheses, The International Journal of Prosthodontics 26 (2013), 343-349.

[2] E. Evangelinaki, D. Tortopidis, E. Kontonasaki, T. Fragou, C. Gogos and P. Koidis, Effect of a crown ferrule on the fracture strength of endodontically treated canines restored with fiber posts and metal-ceramic or all-ceramic crowns, The International Journal of Prosthodontics 26 (2013), 384-387.

[3] R.R. Barcellos, D.P. Correia, A.P. Farina, M.F. Mesquita, C.C. Ferraz and D. Cecchin, Fracture resistance of endodontically treated teeth restored with intra-radicular post: The effects of post system and dentine thickness, Journal of Biomechanics 46 (2013), 2572-2577.

[4] J. Juloski, D. Apicella and M. Ferrari, The effect of ferrule height on stress distribution within a tooth restored with fibre posts and ceramic crown: A finite element analysis, Dental Materials: Official Publication of the Academy of Dental Materials 30 (2014), 1304-1315.

[5] M.G. Roscoe, P.Y. Noritomi, V.R. Novais and C.J. Soares, Influence of alveolar bone loss, post type, and ferrule presence on the biomechanical behavior of endodontically treated maxillary canines: Strain measurement and stress distribution, The Journal of Prosthetic Dentistry 110 (2013), 116-126.

[6] D. Adyani-Fard, T.S. Kim and P. Eickholz, Interproximal bone loss at contra-lateral teeth with and without root canal filling in periodontitis patients, Journal of Clinical Periodontology 38 (2011), 269-275.

[7] H.M. Abou El Nasr and K.G. Abd El Kaden, Dentinal damage and fracture resistance of oval roots prepared with single-file systems using different kinematics, Journal of Endodontics 40 (2014), 849-851.

[8] O.S. Gbadebo, D.M. Ajayi, O.O. Oyekunle and P.O. Shaba, Randomized clinical study comparing metallic and glass fiber post in restoration of endodontically treated teeth, Indian Journal of Dental Research: Official Publication of Indian Society for Dental Research 25 (2014), 58-63.

[9] G. Sterzenbach, A. Franke and M. Naumann, Rigid versus flexible dentine-like endodontic posts-clinical testing of a biomechanical concept: Seven-year results of a randomized controlled clinical pilot trial on endodontically treated abutment teeth with severe hard tissue loss, Journal of Endodontics 38 (2012), 1557-1563.

[10] P.P. Shetty, R. Meshramkar, K.N. Patil and R.K. Nadiger, A finite element analysis for a comparative evaluation of stress with two commonly used esthetic posts, European Journal of Dentistry 7 (2013), 419-422.

[11] F.A. Alharbi, D. Nathanson, S.M. Morgano and N.Z. Baba, Fracture resistance and failure mode of fatigued endodontically treated teeth restored with fiber-reinforced resin posts and metallic posts in vitro, Dental Traumatology 30 (2014), 317-325. 
[12] M. Vano, C. Goracci, F. Monticelli, F. Tognini, M. Gabriele, F.R. Tay, et al., The adhesion between fibre posts and composite resin cores: The evaluation of microtensile bond strength following various surface chemical treatments to posts, International Endodontic Journal 39 (2006), 31-39.

[13] S. Toksavul, M. Zor, M. Toman, M.A. Gungor, I. Nergiz and C. Artunc, Analysis of dentinal stress distribution of maxillary central incisors subjected to various post-and-core applications, Operative Dentistry 31 (2006), 89-96.

[14] M. Borba, M.D. de Araujo, E. de Lima, H.N. Yoshimura, P.F. Cesar, J.A. Griggs, et al., Flexural strength and failure modes of layered ceramic structures, Dental Materials 27 (2011), 1259-1266.

[15] F.S. Schwindling, T. Hartmann, D. Panagidis, J. Krisam, S. Rues and M. Schmitter, In vitro investigation on extensively destroyed vital teeth: Is fracture force a limiting factor for direct restoration? Journal of Oral Rehabilitation 41 (2014), 920-927.

[16] B. Dejak and A. Mlotkowski, Finite element analysis of strength and adhesion of cast posts compared to glass fiber-reinforced composite resin posts in anterior teeth, The Journal of Prosthetic Dentistry 105 (2011), 115-126.

[17] M. Toman, S. Toksavul, M. Sarikanat, I. Nergiz and P. Schmage, Fracture resistance of endodontically treated teeth: effect of tooth coloured post material and surface conditioning, The European Journal of Prosthodontics and Restorative Dentistry 18 (2010), 23-30.

[18] S.F. Chuang, P. Yaman, A. Herrero, J.B. Dennison and C.H. Chang, Influence of post material and length on endodontically treated incisors: An in vitro and finite element study, The Journal of Prosthetic Dentistry 104 (2010), 379-388

[19] J. Bijelic, S. Garoushi, P.K. Vallittu and L.V. Lassila, Short fiber reinforced composite in restoring severely damaged incisors, Acta Odontologica Scandinavica 71 (2013), 1221-1231.

[20] F. Zicari, B. Van Meerbeek, E. Debels, E. Lesaffre and I. Naert, An up to 3-year controlled clinical trial comparing the outcome of glass fiber posts and composite cores with gold alloy-based posts and cores for the restoration of endodontically treated teeth, The International Journal of Prosthodontics 24 (2011), 363-372.

[21] N. Beck, F. Graef, M. Wichmann and M. Karl, In vitro fracture resistance of copy-milled zirconia ceramic posts, The Journal of Prosthetic Dentistry 103 (2010), 40-44.

[22] N. Bittner, T. Hill and A. Randi, Evaluation of a one-piece milled zirconia post and core with different post-and-core systems: An in vitro study, The Journal of Prosthetic Dentistry 103 (2010), 369-379. 